\title{
Infectious

\section{Bacterial Derived Carbohydrates Bind Cyr1 and Trigger Hyphal Growth in Candida albicans}

\author{
Jason M. Burch, ${ }^{\dagger}$ Siavash Mashayekh, ${ }^{\dagger}$ Dennis D. Wykoff, ${ }^{\dagger}$ and Catherine L. Grimes ${ }^{*},, \S_{\odot}$ \\ ${ }^{\dagger}$ Department of Chemistry and Biochemistry, University of Delaware, Newark, Delaware 19716, United States \\ ${ }^{\ddagger}$ Department of Biology, Villanova University, Villanova, Pennsylvania 19085, United States \\ ${ }^{\S}$ Department of Biological Sciences, University of Delaware, Newark, Delaware 19716, United States
}

\section{Supporting Information}

ABSTRACT: The dimorphic yeast Candida albicans is the most common pathogenic fungus found in humans. While this species is normally commensal, a morphological switch from budding yeast to filamentous hyphae allows the fungi to invade epithelial cells and cause infections. The phenotypic change is controlled by the adenylyl cyclase, Cyr1. Interestingly, this protein contains a leucine-rich repeat (LRR) domain, which is commonly found in innate immune receptors from plants and animals. A functional and pure LRR domain was obtained in high yields from E. coli expression. Utilizing a surface plasmon resonance assay, the LRR was found to bind diverse bacterial derived carbohydrates with high affinity. This domain is capable of binding fragments of peptidoglycan, a carbohydrate polymer component of the bacterial cell wall, as well as anthracyclines produced by Streptomyces, leading to hyphae formation. These findings add another dimension to the human microbiome, taking into account yeast-bacteria interactions that occur in the host.

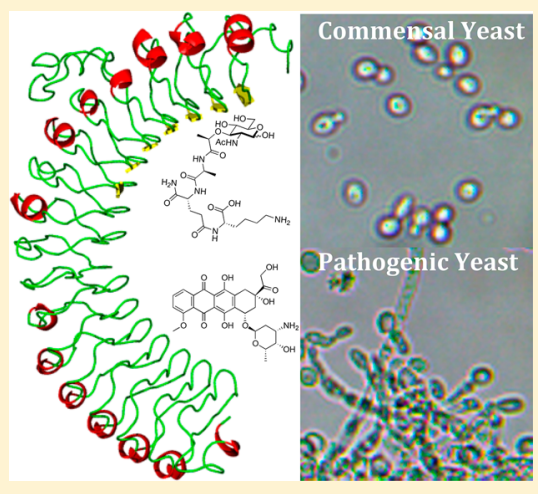

KEYWORDS: C. albicans, anthracycline, peptidoglycan, hyphae formation, leucine-rich-repeat domain

$\mathrm{T}$ he human body plays host to trillions of microorganisms collectively referred to as the microbiome. It has been reported that there are as many bacteria as human cells in the average individual. ${ }^{1}$ While most literature examining the microbiome focuses on these bacterial species, there is a smaller yet no less important population of fungal species, the "mycobiome", present in all individuals. ${ }^{2}$ Similar to host immune cells, these fungi can recognize changes in the microbiome composition often leading to disease states. ${ }^{3}$ Candida albicans is perhaps the most recognizable fungal infection, as either oral candidiasis (or "thrush") or vaginal candidiasis commonly referred to as a "yeast infection", which affects nearly $75 \%$ of women at least once in their lifetime. ${ }^{4}$ Candida infections can also enter the bloodstream, where patient outcomes are much worse. Candida species are responsible for $9 \%$ of hospital acquired bloodstream infections with mortality rates at approximately $20 \% .^{5,6}$ To effectively treat these infections, it is important to understand how these fungi are turning pathogenic.

C. albicans is a dimorphic fungus capable of growing as budding yeast or as filamentous hyphae. ${ }^{7}$ It is the hyphae form which allows C. albicans to form biofilms ${ }^{8}$ and become invasive, by either endocytosis by the host cell or active penetration of the plasma membrane by the hyphae.' Several pathways including the cAMP-PKA, MAPK, cell cycle arrest, and $\mathrm{pH}$ pathways regulate the morphogenesis of $C$. albicans. ${ }^{10}$ The cAMP-PKA pathway is of central importance, as Rocha et al. have demonstrated that knocking out the $\mathrm{CaCDC} 35$ gene (also known as CaCYR1), which encodes the adenylyl cyclase, Cyr1, prevents hyphae formation in C. albicans. ${ }^{11}$ Cyr1 responds to a number of signals including G-protein receptors, RAS proteins, elevated temperatures, $\mathrm{CO}_{2}$ levels, quorum sensing molecules, farnesol, homoserine lactone, and serum. ${ }^{12}$ Although serum has been known since the 1950 s to elicit hyphae formation, ${ }^{13}$ it was not until 2008 that the compound responsible for hyphal growth was identified as fragments of peptidoglycan, ${ }^{14}$ the carbohydrate polymer which forms the bacterial cell wall. ${ }^{15}$ This proved especially interesting, as studies have indicated that C. albicans infections increase with fluctuations in microbiome composition, especially those caused by antibiotic treatment. ${ }^{16-20}$ Cyr 1 contains a leucine-rich repeat (LRR) domain, an evolutionary conserved motif common in many innate immune receptors found in both mammals and plants. ${ }^{21}$ Like many of these receptors, Cyr1 is able to bind to peptidoglycan fragments, specifically muramyl dipeptide (MDP) (1, Figure 1). ${ }^{14} \mathrm{Xu}$ et al. $^{14}$ demonstrated this interaction using a streptavidin pull down assay employing biotinylated MDP. This work provided a molecular identity to the hyphae inducing agent in serum.

Peptidoglycan is a polymer of the carbohydrates, $\mathrm{N}$-acetylglucosamine and $\mathrm{N}$-acetyl-muramic acid. ${ }^{15}$ The latter of these is used exclusively in bacterial peptidoglycan, thus making it unique to bacteria. Interestingly, fragments of the bacterial cell

Special Issue: The Microbiome

Received: September 15, 2017

Published: October 17, 2017 


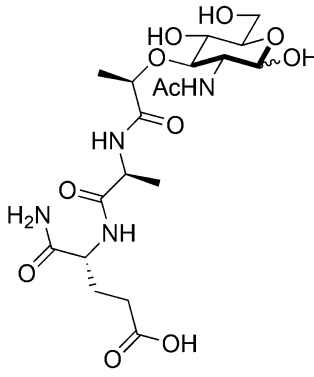

Muramyl Dipeptide (MDP)<smiles>COc1cccc2c1C(=O)c1c(O)c3c(c(O)c1C2=O)C[C@@](O)(C(=O)CO)C[C@H](OC1C[C@H](N)[C@H](O)[C@H](C)O1)O3</smiles>

Doxorubicin

2

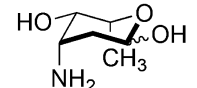

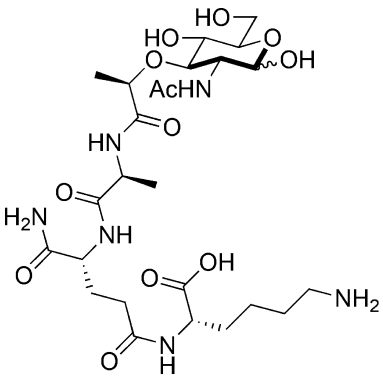

Muramyl Tripeptide (MTP)

Figure 1. Bacterial derived carbohydrates. A diverse set of carbohydrates were chosen to test for their ability to induce hyphae formation in C. albicans. MDP (1) and MTP (4) are fragments of the bacterial peptidoglycan. Daunosamine (3) is the sugar moiety of the Stretomyces peucetius natural product and chemotherapeutic agent doxorubicin $(2)$.
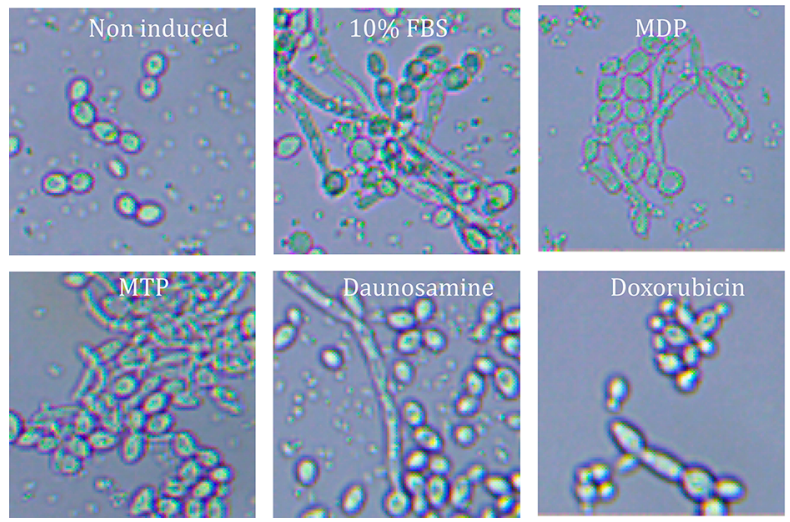

\begin{tabular}{lr}
\hline \multicolumn{2}{c}{ Percent } \\
Hyphae \\
\hline Non Induced & $\mathbf{6 . 1 4} \%$ \\
10\% FBS & $\mathbf{3 6 . 7 7 \%}$ \\
\hline MDP & $\mathbf{2 9 . 1 3 \%}$ \\
\hline MTP & $\mathbf{4 1 . 4 3 \%}$ \\
\hline Daunosamine & $\mathbf{6 . 8 3} \%$ \\
\hline Doxorubicin & $\mathbf{7 . 4 9} \%$ \\
\hline
\end{tabular}

Figure 2. Bacterial derived carbohydrates elicit hyphae formation in C. albicans. $1 \mathrm{~mL}$ C. albicans cultures were grown overnight at $37{ }^{\circ} \mathrm{C}$ in $\mathrm{YPD}$ media in the presence of $10 \%$ fetal bovine serum (FBS) or $1 \mathrm{mM}$ of the indicated compound. Cells were scored for hyphal growth and reported as a percentage. Specific details regarding scoring cells can be found in the Methods.

Scheme 1. Synthesis of Muramyl Tripeptide (Lysine) ${ }^{a}$<smiles>CC(N)C(=O)NC(CCC(=O)NC(CCCCNC(C)(C)C)C(N)=O)C(=O)OCc1ccccc1</smiles>
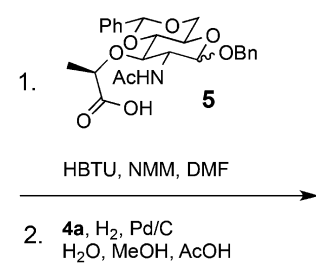

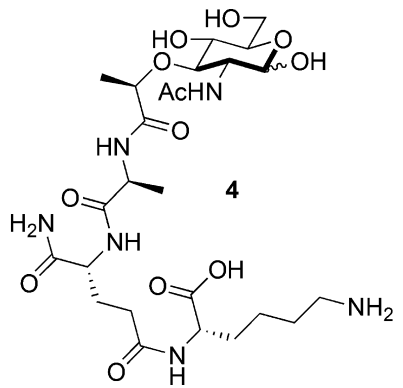

${ }^{a}$ Reagents and conditions: (a) 1. HBTU, N-methylmorpholine, DMF, 2. protected tripeptide, 64\%; (b) Pd/C, $\mathrm{H}_{2} \mathrm{O}, \mathrm{MeOH}, \mathrm{AcOH}, 95 \%$.

wall are not the only carbohydrate containing compounds known to affect the morphology of C. albicans. In 2010, Kwok et al. showed that DNA topoisomerase inhibitors, specifically anthracyclines, altered the morphology of C. albicans. ${ }^{22}$ The ability of anthracyclines, such as doxorubicin (2, Figure 1), to induce $C$. albicans hyphae formation is intriguing as the compounds are natural products produced by Streptomyces peucetius and contain the L-sugar, daunosamine (3, Figure 1). Chemotherapy has been linked to an increased risk for systemic Candida infections in cancer patients. ${ }^{23}$ We hypothesized that the bacterial derived carbohydrate could play a role in this phenotype and sought to establish a robust biochemical assay to probe the importance of the carbohydrate.
In this work, we expanded the scope of bacterial derived carbohydrates capable of eliciting a hyphae response in C. albicans. Further, we report an improved method for expressing and purifying the LRR of Cyr1 in Escherichia coli, resulting in high yields and removing contaminating peptidoglycan fragments. Finally, using a surface plasma resonance (SPR) assay, previously developed in our lab to characterize the NOD2-MDP interaction, ${ }^{24-26}$ we characterize the ability of the LRR domain to bind a subset of bacterial derived carbohydrates.

\section{RESULTS AND DISCUSSION}

A binding assay for a LRR containing protein, Nod2, and a peptidoglycan fragment was established in our laboratory. ${ }^{24}$ In 
A

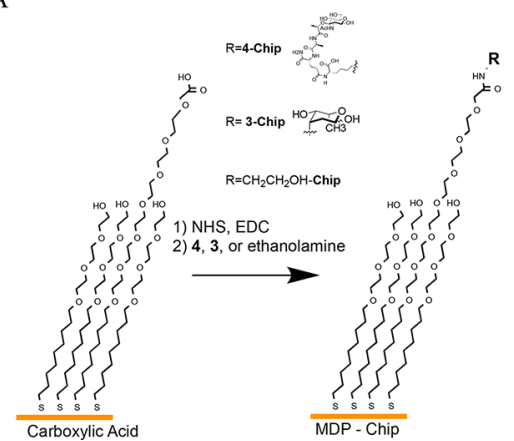

B

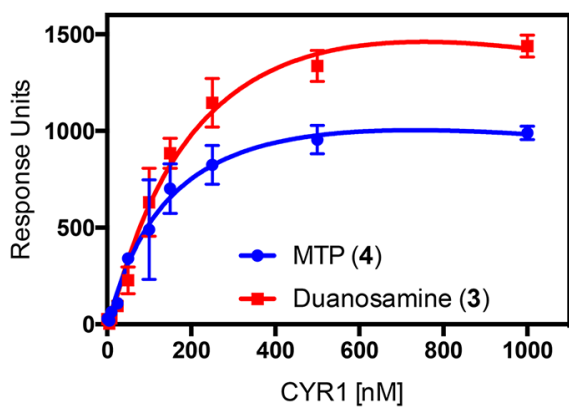

Figure 3. SPR assay to determine LRR affinity for MTP and daunosamine. (A) After activation of the SAMs layer using NHS and EDC, the compounds were applied to the chip in their respective lane. Coupling resulted in the formation of an amide bond on the lysine residue of MTP (4) or the 3 position of daunosamine (3). A control lane of ethanolamine was applied to account for nonspecific binding. (B) A binding curve was generated from the sensograms produced by the SPR assay. LRR bound to MTP (4) with a $K_{\mathrm{D}}$ of $176 \pm 68 \mathrm{nM}$ and to daunosamine (3) with a $K_{\mathrm{D}}$ of $287 \pm 88 \mathrm{nM}$.

this assay, the peptidoglycan fragment was linked via an amine handle, which was installed on the carbohydrate backbone. However, in the initial binding experiments between MDP and Cyr1, a biotin affinity handle was added to the peptide portion of the peptidoglycan fragments. ${ }^{14}$ These data suggest that a quantitative binding assay could support modifications on the peptide. Here, a synthesis of a new peptidoglycan fragment, muramyl tripeptide (lysine) (MTP) (4, Figure 1), was developed. Gram-positive bacteria naturally incorporate a lysine at the third position of the pentapeptide in their peptidoglycans. ${ }^{15}$ It was reasoned that the amine on the lysine would serve as an attachment point for the SPR assay (Figure 2A). Boons and co-workers established a novel chemistry for producing such fragments on a solid phase. ${ }^{27}$ Recently, we have developed robust solution chemistry to access amino versions of peptidoglycan fragments which utilizes a benzyl protected carbohydrate (5, Scheme 1). ${ }^{26}$ Here, a tripeptide (7, Scheme $1)$, which contains benzyl protecting groups, was synthesized using solution based chemistry (see the Supporting Information for synthetic details; Supplemental Scheme 1). 7 was coupled to 5 using coupling reagents to minimize epimerization, and the resulting product was globally deprotected using catalytic hydrogenation to yield 4, a novel MTP whose tripeptide contains L-alanine, D-isoglutamine, and L-lysine. As in many peptidoglycans found in nature, the isoglutamine in $\mathbf{4}$ is linked to the lysine via the side chain as opposed to the peptide backbone and the $\alpha$-carboxyl group of glutamic acid is amidated. $^{28}$ Boons and co-workers have shown that MTPs retained the ability to activate an immune response in humans; ${ }^{27}$ however, it was not known if MTPs would activate the hyphal response in yeast.

Compound 4 was tested for the ability to induce hyphae growth in C. albicans (Figure 2) along with a known activator, $\operatorname{MDP}$ (1, Figure 1). 1 induced hyphal formation, in agreement with the previously published results. ${ }^{14}$ However, 4 proved to be more potent in activating hyphae growth, while also appearing to initiate biofilm formation (Supplemental Figure $1)$. These data show that MTPs are capable of activating a response in yeast.

In addition to probing peptidoglycan carbohydrates, the ability of doxorubicin (2, Figure 1), a glycosylated anthracycline produced by bacteria, and its associated carbohydrate, daunosamine (3, Figure 1), to activate hyphal growth was screened. 2 triggered a slight increase in hyphal growth but also caused cell death, which can be expected as $\mathbf{2}$ is a topoisomerase inhibitor. $^{22}$ Interestingly, 3, which is only the carbohydrate component, was also able to mildly induce hyphae formation without the associated cell death seen with the parent compound, 2. These results suggest that the carbohydrates from peptidoglycan and bacterial derived small molecules are important for inducing hyphal growth.

Intrigued by the results of the hyphal growth assay, the ability for the carbohydrates to bind to Cyr1 was assessed. To accomplish this, a robust purification of the LRR domain of Cyr1 had to be developed. Previous work from $\mathrm{Xu}$ et al. established purification of a GST-LRR fusion protein, which was used to demonstrate LRR-MDP binding via a biotinstreptavidin pull down assay and subsequently resolved via Western blot analysis. ${ }^{14}$ However, in our hands, this method did not give high enough yields of protein to use in the desired quantitative binding assay. A hexahistidine-tagged maltosebinding protein (MBP) fusion protein, which is known to increase protein solubility, was added to the N-terminus. ${ }^{29}$ Despite the addition of the MBP tag, the protein remained largely insoluble when expressed in E. coli. Alterations in expression and lysis conditions were not successful in increasing yields of the soluble fusion protein. Cyrl-LRR could be precipitating as it is expressed in the presence of a known ligand, peptidoglycan fragments. Therefore, a denaturationrefolding technique was employed to extract the fusion protein from inclusion bodies produced during protein expression. These conditions would ensure that any bound peptidoglycan fragment/ligand would be released. Using this method, MBP fused to Cyrl's LRR was obtained in good yield $(10 \mathrm{mg} / \mathrm{L}$ of expression) with $\sim 90 \%$ purity (Supplemental Figure $2 \mathrm{~A}$ ). The protein showed a narrow range of stability as it aggregated when dialyzed into a buffer below pH 6.0 (Supplemental Figure 2B), which was important for developing the SPR binding assay. The refolding of the protein was confirmed by circular dichroism (CD). Minima at 208 and $222 \mathrm{nM}$ indicated that the fusion protein contained high $\alpha$-helical character, consistent with the structure of the LRR domain of homologous proteins. ${ }^{30}$ To ensure that secondary structure was conserved during the refolding process, a CD spectrum of MBP-LRR that was expressed in the soluble fraction was obtained and compared to the spectrum of the refolded protein (Supplemental Figure 3). Both spectra show a similar minimum, indicating the protein was able to refold with the proper secondary structure. Protein identity was further confirmed by LC-MS/MS (Supplemental Figure 4). Using a MBP tag and a 

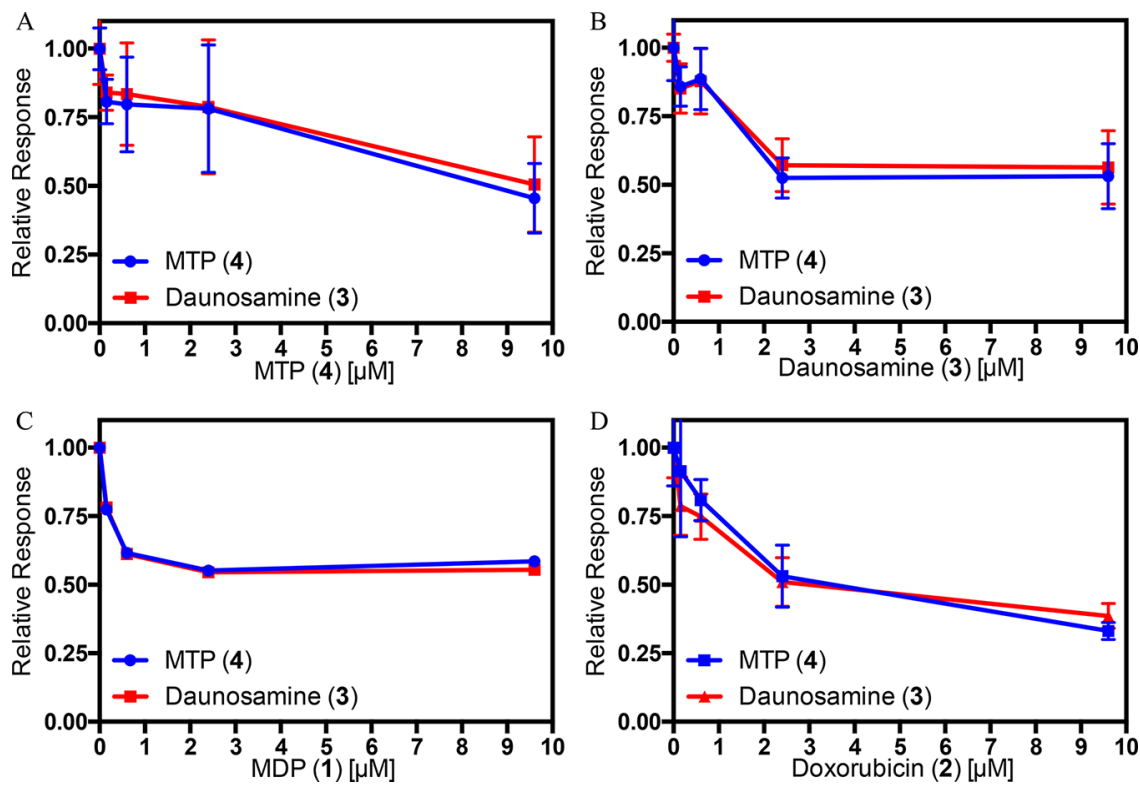

Figure 4. SPR competition assay. 0.15-9.6 $\mu \mathrm{M}$ of 1-4 were preincubated with $150 \mathrm{nM}$ Cyrl-LRR. The protein mixture was applied to the SPR chip, and the response was compared to apo protein. (A) Preincubation with 4 reduced binding to 3 by $49.5 \%$ and 4 by $54.5 \%$, (B) 3 reduced binding to 3 by $43.7 \%$ and 4 by $49.5 \%$, (C) 1 reduced binding to 3 by $44.6 \%$ and 4 by $41.5 \%$, and (D) 2 reduced binding to 3 by $61.4 \%$ and 4 by $66.9 \%$.

denature/renature purification procedure, Cyrl's LRR was obtained in large quantity and high purity.

With the purified protein in hand, an assay was developed to determine if the compounds capable of inducing hyphae growth (Figure 2) could bind to the LRR domain of Cyr1. Our laboratory has developed a surface plasmon resonance (SPR) assay, $^{24,26}$ which has been used to measure the binding of bacterial peptidoglycan fragments to the LRR domain of the innate immune receptor NOD2. ${ }^{25}$ This assay was modified to determine the affinity of the LRR from Cyr1 to the bacterial derived carbohydrates. Briefly, the SPR analysis employed involves covalently attaching a ligand of interest to a selfassembled monolayer (SAM) attached to a gold chip (Figure 3A). The constructed SAM contained MTP (4), daunosamine (3), and ethanolamine; this chip was utilized to probe the LRR's ability to bind the various carbohydrates. The purified MBP-LRR was applied to the ligand-modified surface, and changes in refraction index were monitored in order to generate a SPR sensogram (Supplemental Figure 5). Using this SPR assay, it was determined that the LRR bound to 4 with a $K_{\mathrm{D}}$ of $176 \pm 68 \mathrm{nM}$ and to 3 with a $K_{\mathrm{D}}$ of $287 \pm 88 \mathrm{nM}$ (Figure $3 \mathrm{~B}$ ). These data demonstrate that the LRR is capable of binding diverse carbohydrates with high affinity. To verify that the LRR is responsible for the binding event, the free MBP tag was purified as a control and applied to the chip. The free MBP tag was unable to bind either compound (Supplemental Figure 6) suggesting that the observed binding is specific to the LRR.

To validate the observed $K_{\mathrm{D}}$ was due to Cyr1-LRR affinity for the carbohydrates and not the result of nonspecific absorption to the chip, a competition assay was performed. Cyr1-LRR was preincubated with increasing concentrations of 3 or 4, ranging from the approximate $K_{\mathrm{D}}$ up to 64 -fold the $K_{\mathrm{D}}$. The protein was applied to the SPR chip, and the response was recorded and compared to the response levels of the apo Cyr1LRR. Preincubation with 4 reduced the binding response to 3 and 4 by $49.5 \%$ and $54.5 \%$, respectively (Figure 4A). Preincubation with 3 was as effective with response to 3 and
4 at $56.3 \%$ and $50.5 \%$ of apo Cyr1-LRR binding levels (Figure 4B). With confirmation that the SPR signals were the result of Cyr1-LRR specifically binding to the carbohydrates, the competition experiment was repeated with $\mathbf{1}$ and $\mathbf{2}$ to probe their ability to bind Cyrl-LRR and inhibit binding to the immobilized carbohydrates. MDP (1, Figure 1) was capable of reducing binding of Cyr1-LRR to 3 and 4 to $55.4 \%$ and $58.5 \%$ of apo levels, respectively (Figure 4C). Preincubation with doxorubicin (2, Figure 1) reduced responses to 3 and 4 by $61.4 \%$ and $66.9 \%$, respectively (Figure 4D). These data demonstrate the Cyrl's LRR has a versatile binding site. Cyr1 contains 14 LRRs, and the data indicate a single binding site within this domain, as $\mathbf{1 - 4}$ are capable of reducing binding to peptidoglycan or anthracycline associated carbohydrates. To ensure the decrease in binding affinity was not a result of nonspecific interactions, CYR1-LRR was incubated with alanine, glutamine, or chloramphenicol. These compounds did not decrease the response compared to the apo Cyr1-LRR (Supplemental Figure 7). However, in order to fully appreciate Cyr1-LRR's carbohydrate binding pocket, future experiments, either mutational in nature or synthetic (i.e., photoactivatable cross-linkers), are needed to determine the molecular makeup of Cyrl's carbohydrate binding residues.

The development of a robust E. coli based expression and refolding purification protocol grants access to sufficient quantities of the LRR domain of Cyr1, free from contaminating natural ligands that are present when the protein is expressed in a bacterial host. Using a quantitative binding assay, Cyrl's LRR domain was found to bind a diverse range of carbohydrates from bacterial sources with high affinity, highlighting the importance of the interactions between yeast and bacteria in the human microbiome. The development of this assay will allow for the potential identification of compounds that inhibit carbohydrate binding and subsequent hyphae formation, ultimately, leading to novel therapeutics, which can be coadministered with antibiotics or chemotherapeutics to prevent systemic C. albicans infections. 


\section{METHODS}

For full experimental details regarding materials, synthetic protocols, protein expression and purification, SPR procedures, and the hyphal activation assay, refer to the Supporting Information.

Synthesis of MTP. Standard peptide coupling conditions were used to couple the tripeptide to the protected muramic acid. Global deprotection was performed using $\mathrm{Pd} / \mathrm{C}$ and $\mathrm{H}_{2}$. MTPs were purified using a Waters auto purification system. For detailed synthetic protocols, including ${ }^{1} \mathrm{H}$ and ${ }^{13} \mathrm{C}$ NMR and high-resolution mass spectra, please see the Supporting Information.

Hyphal Growth Assay. Ten $\mathrm{mL}$ of yeast peptone dextrose (YPD) was inoculated with C. albicans (SC5314) from a glycerol stock stored at $-80{ }^{\circ} \mathrm{C}$. The culture was grown overnight at $30{ }^{\circ} \mathrm{C}$. The cells were separated into $0.9 \mathrm{~mL}$ aliquots, and $100 \mu \mathrm{L}$ of sterile $\mathrm{H}_{2} \mathrm{O}$ or $10 \mathrm{mM}$ MTP (4), MDP (1), daunosamine (3), or doxorubicin (2) was added. Cultures were incubated overnight at $37{ }^{\circ} \mathrm{C}$ while shaking at $200 \mathrm{rpm}$. Aliquots were taken and imaged at $40 \times$ magnification. Images containing approximately 100 cells were blindly scored for hyphal growth. Hyphal growth was determined by dividing cells displaying hyphae characteristics by total cells. Percentages reported are the average of two independent trials.

Expression and Purification of Constructs. The MBPLRR was expressed at $30{ }^{\circ} \mathrm{C}$ until an optical density $(600 \mathrm{~nm})$ of $0.6-0.8$ was reached. Protein expression was induced by the addition of IPTG to a concentration of $1.0 \mathrm{mM}$ overnight at 18 ${ }^{\circ} \mathrm{C}$. A pellet from $1 \mathrm{~L}$ of cells was resuspended and lysed using sonication. The inclusion body pellet was washed and denatured. A clarified inclusion body suspension was applied to the $5 \mathrm{~mL}$ HisTrap HP column (GE LifeSciences) on a NGC Quest 10 chromatography system (Bio-Rad). After washing with low amounts of imidazole, the protein was eluted with high concentrations of imidazole. Fractions containing the MBP-LRR were pooled and dialyzed overnight into refolding buffer with high concentrations of arginine. Refolded protein was dialyzed into Biacore assay buffer. Protein concentration was determined by absorbance at $280 \mathrm{~nm}\left(\varepsilon=91595 \mathrm{M}^{-1}\right.$ $\left.\mathrm{cm}^{-1}\right)$. For detailed protein purification protocols, including SDS-PAGE images, CD spectrum, and mass spectra, please see the Supporting Information.

Surface Plasmon Resonance Assay. Methods for preparing the chip, mixed-SAM, and SPR assay conditions were adapted from Grimes et al. ${ }^{24}$ The BIAcore 3000 instrument was used for all SPR assays described. Full experimental details and raw sensograms are presented in the Supporting Information.

\section{ASSOCIATED CONTENT}

\section{S Supporting Information}

The Supporting Information is available free of charge on the ACS Publications website at DOI: 10.1021/acsinfecdis.7b00154.

Supplemental methods such as materials, expression and purification of LRR-CRY1, SPR conditions, hyphal growth assay, synthetic procedures, and NMR data (PDF)

\section{AUTHOR INFORMATION}

\section{Corresponding Author}

*E-mail: cgrimes@udel.edu.
ORCID

Catherine L. Grimes: 0000-0002-0586-2879

\section{Author Contributions}

The study was designed by J.M.B., D.D.W., and C.L.G.; J.M.B. and S.M. conducted the experiments. All authors analyzed the data. J.M.B. and C.L.G. wrote the manuscript, and all authors have read and given approval to the final version.

\section{Notes}

The authors declare no competing financial interest.

\section{ACKNOWLEDGMENTS}

The authors are thankful for support from the Delaware COBRE program, supported by a grant from the National Institute of General Medical Sciences (NIGMS 1 P30 GM110758 and 1 P20 GM104316-01A1) from the National Institutes of Health. Acknowledgement is gratefully made to the National Science Foundation (CAREER CHE 1554967) for support of this research. We thank Daniel Scanlon, James Melnyk, and Amy Schaefer for assistance in the experimental design. We thank Kristen DeMeester for assistance with formatting the NMR spectra and critically reading the Supporting Information. We thank the Zondlo lab for use of their CD instrument, the Rozovsky lab for the modified pMALc5-x plasmid, and the mass spectrometry facility at the University of Delaware.

\section{ABBREVIATIONS}

MDP, muramyl dipeptide; LRR, leucine-rich repeat; SPR, surface plasmon resonance; CD, circular dichroism; SAM, selfassembled monolayer; MTP, muramyl tripeptide; YPD, yeast peptone dextrose; FBS, fetal bovine serum

\section{REFERENCES}

(1) Sender, R., Fuchs, S., and Milo, R. (2016) Revised Estimates for the Number of Human and Bacteria Cells in the Body. PLoS Biol. 14 (8), e1002533.

(2) Huffnagle, G. B., and Noverr, M. C. (2013) The emerging world of the fungal microbiome. Trends Microbiol. 21 (7), 334-41.

(3) Underhill, D. M., and Iliev, I. D. (2014) The mycobiota: interactions between commensal fungi and the host immune system. Nat. Rev. Immunol. 14 (6), 405-16.

(4) Sobel, J. D. (1997) Vaginitis. N. Engl. J. Med. 337 (26), 1896903.

(5) Wisplinghoff, H., Bischoff, T., Tallent, S. M., Seifert, H., Wenzel, R. P., and Edmond, M. B. (2004) Nosocomial bloodstream infections in US hospitals: analysis of 24,179 cases from a prospective nationwide surveillance study. Clin. Infect. Dis. 39 (3), 309-17.

(6) Morgan, J., Meltzer, M. I., Plikaytis, B. D., Sofair, A. N., HuieWhite, S., Wilcox, S., Harrison, L. H., Seaberg, E. C., Hajjeh, R. A., and Teutsch, S. M. (2005) Excess mortality, hospital stay, and cost due to candidemia: a case-control study using data from population-based candidemia surveillance. Infect Control Hosp Epidemiol 26 (6), 540-7.

(7) Soll, D. R. (1992) High-frequency switching in Candida albicans. Clin. Microbiol. Rev. 5 (2), 183-203.

(8) Sudbery, P. E. (2011) Growth of Candida albicans hyphae. Nat. Rev. Microbiol. 9 (10), 737-48.

(9) Zhu, W., and Filler, S. G. (2010) Interactions of Candida albicans with epithelial cells. Cell. Microbiol. 12 (3), 273-82.

(10) Shapiro, R. S., Robbins, N., and Cowen, L. E. (2011) Regulatory circuitry governing fungal development, drug resistance, and disease. Microbiology and molecular biology reviews: MMBR 75 (2), 213-67.

(11) Rocha, C. R., Schroppel, K., Harcus, D., Marcil, A., Dignard, D., Taylor, B. N., Thomas, D. Y., Whiteway, M., and Leberer, E. (2001) Signaling through adenylyl cyclase is essential for hyphal growth and 
virulence in the pathogenic fungus Candida albicans. Mol. Biol. Cell 12 (11), 3631-43.

(12) Wang, Y. (2013) Fungal adenylyl cyclase acts as a signal sensor and integrator and plays a central role in interaction with bacteria. PLoS Pathog. 9 (10), e1003612.

(13) Reynolds, R., and Braude, A. (1956) The filament inducing property of blood for Candida albicans: its nature and significance. Clin. Res. Proc. 4, 40.

(14) Xu, X. L., Lee, R. T., Fang, H. M., Wang, Y. M., Li, R., Zou, H., Zhu, Y., and Wang, Y. (2008) Bacterial peptidoglycan triggers Candida albicans hyphal growth by directly activating the adenylyl cyclase Cyr1p. Cell Host Microbe 4 (1), 28-39.

(15) Rogers, H. J. (1974) Peptidoglycans (mucopeptides): structure, function, and variations. Ann. N. Y. Acad. Sci. 235 (0), 29-51.

(16) Mason, K. L., Erb Downward, J. R., Mason, K. D., Falkowski, N. R., Eaton, K. A., Kao, J. Y., Young, V. B., and Huffnagle, G. B. (2012) Candida albicans and bacterial microbiota interactions in the cecum during recolonization following broad-spectrum antibiotic therapy. Infect. Immun. 80 (10), 3371-80.

(17) Forster, T. M., Mogavero, S., Drager, A., Graf, K., Polke, M., Jacobsen, I. D., and Hube, B. (2016) Enemies and brothers in arms: Candida albicans and gram-positive bacteria. Cell. Microbiol. 18 (12), 1709-1715.

(18) Bisschop, M. P., Merkus, J. M., and Van Cutsem, J. (1985) The growth-promoting activity of vaginal fluid for Candida albicans (and the problem of enhanced susceptibility to vaginal candidosis). Eur. J. Obstet. Gynecol. Reprod. Biol. 20 (2), 107-12.

(19) Bisschop, M. P., Merkus, J. M., and Van Cutsem, J. (1985) The influence of antibiotics on the growth of Candida albicans in the vagina: an experiment with vaginal fluid. Eur. J. Obstet. Gynecol. Reprod. Biol. 20 (2), 113-9.

(20) Erb Downward, J. R., Falkowski, N. R., Mason, K. L., Muraglia, R., and Huffnagle, G. B. (2013) Modulation of post-antibiotic bacterial community reassembly and host response by Candida albicans. Sci. Rep. 3, 2191.

(21) Inohara, Chamaillard, McDonald, C., and Nunez, G. (2005) NOD-LRR proteins: role in host-microbial interactions and inflammatory disease. Annu. Rev. Biochem. 74, 355-83.

(22) Kwok, S. C., Schelenz, S., Wang, X., and Steverding, D. (2010) In vitro effect of DNA topoisomerase inhibitors on Candida albicans. Med. Mycol. 48 (1), 155-60.

(23) Teoh, F., and Pavelka, N. (2016) How Chemotherapy Increases the Risk of Systemic Candidiasis in Cancer Patients: Current Paradigm and Future Directions. Pathogens 5 (1), 6.

(24) Grimes, C. L., Ariyananda, L. D. Z., Melnyk, J. E., and O'Shea, E. K. (2012) The innate immune protein Nod2 binds directly to MDP, a bacterial cell wall fragment. J. Am. Chem. Soc. 134 (33), 13535-7.

(25) Lauro, M. L., D’Ambrosio, E. A., Bahnson, B. J., and Grimes, C. L. (2017) Molecular Recognition of Muramyl Dipeptide Occurs in the Leucine-rich Repeat Domain of Nod2. ACS Infect. Dis. 3 (4), 264-270.

(26) Schaefer, A. K., Melnyk, J. E., Baksh, M. M., Lazor, K. M., Finn, M. G., and Grimes, C. L. (2017) Membrane Association Dictates Ligand Specificity for the Innate Immune Receptor NOD2. ACS Chem. Biol. 12, 2216.

(27) Roychowdhury, A., Wolfert, M. A., and Boons, G. J. (2005) Synthesis and proinflammatory properties of muramyl tripeptides containing lysine and diaminopimelic acid moieties. ChemBioChem 6 (11), 2088-97.

(28) Siewert, G., and Strominger, J. L. (1968) Biosynthesis of the peptidoglycan of bacterial cell walls. XI. Formation of the isoglutamine amide group in the cell walls of Staphylococcus aureus. J. Biol. Chem. 243 (4), 783-790.

(29) Sun, P., Tropea, J. E., and Waugh, D. S. (2011) Enhancing the Solubility of Recombinant Proteins in Escherichia coli by Using Hexahistidine-Tagged Maltose-Binding Protein as a Fusion Partner, in Heterologous Gene Expression in E.coli: Methods and Protocols (Evans, J. T. C., and Xu, M.-Q., Eds.), pp 259-274, Humana Press, Totowa, NJ, DOI: $10.1007 / 978-1-61737-967-3 \_16$.
(30) Istomin, A. Y., and Godzik, A. (2009) Understanding diversity of human innate immunity receptors: analysis of surface features of leucine-rich repeat domains in NLRs and TLRs. BMC Immunol. 10, 48. 